\title{
System Engineering at the MEGARA project
}

\author{
A. Pérez-Calpena, A. ; M.L. García-Vargas ; A. Gil de Paz ; J. Gallego Maestro ; \\ E. Carrasco Licea ${ }^{\mathrm{c}}$; F. Sánchez Moreno ${ }^{\mathrm{d}}$; J. Iglesias-Páramo ${ }^{\mathrm{e}}$ \& MEGARA Team

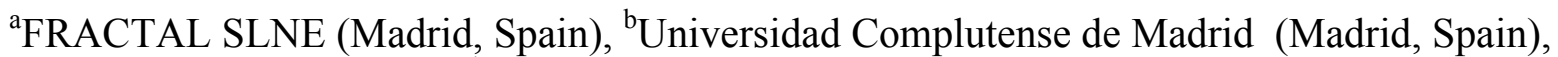 \\ ${ }^{\mathrm{c}}$ INAOE (Puebla, México), ${ }^{\mathrm{d}}$ Universidad Politécnica de Madrid (Madrid, Spain), ${ }^{\mathrm{e}}$ Instituto de \\ Astrofísica de Andalucía IAA-CSIC (Granada, Spain)
}

\begin{abstract}
MEGARA (Multi-Espectrógrafo en GTC de Alta Resolución para Astronomía) is a facility instrument of the 10.4m GTC (La Palma, Spain) working at optical wavelengths that provides both Integral-Field Unit (IFU) and Multi- Object Spectrograph (MOS) capabilities at resolutions in the range $\mathrm{R}=6,000-20,000$. The MEGARA focal plane subsystems are located at one of the GTC focal stations, while the MEGARA refractive VPH based spectrograph is located at one of the Nasmyth platforms. The fiber bundles conduct the light from the focal plane subsystems to the pseudo-slits at the entrance of the spectrograph.

The project is an initiative led by Universidad Complutense de Madrid (Spain) in collaboration with INAOE (Mexico), IAA-CSIC (Spain) and Universidad Politécnica de Madrid (Spain) and is developed under contract with GRANTECAN. The project is carried out by a multidisciplinary and geographically distributed team, which includes the in-kind contributions of the project partners and personnel from several private companies.

The MEGARA system-engineering plan has been tailored to the project and is being applied to ensure the technical control of the project in order to finally meet the science high-level requirements and GTC constrains.
\end{abstract}

Keywords: MEGARA, GTC, System engineering, requirements and specification, technical budgets, RAMS, Product Tree, Interfaces, verification, configuration control, non-conformities, anomalies.

\section{INTRODUCTION}

Systems engineering provides the methodology for developing a system in a structured and orderly manner. This methodology involves following several steps, which include specifying the system requirements, mapping them to the initial needs, and, finally, preparing and carrying out the system verification. The objective of this process is to produce a system that fulfills the initial needs, which is essential for reaching the project success.

A project success occurs when the system meets its objectives and the project is carried out within the cost and schedule assigned to it. In order to accomplish it, the project must be carried out with a good organization. The Systems Engineering Plan provides the means to organize the technical effort of the project.

System Engineering is defined as the interdisciplinary approach governing the total technical effort to transform the initial requirements into the system solution. A System Engineering Plan must describe the approach, techniques, tools, organization, planning and technical effort needed to achieve the project objectives. Systems Engineering considers the whole system life cycle. It means, it is considering from the earliest stages of the project not only to design phase of the system but also the production, integration and operation phases. In this way, the production, maintenance, operation and logistics requirement are taken into account from the beginning.

The System Engineering Plan must be tailored for that particular project. This Plan must include the activities to be performed in each phase of the project, the milestones to be met, the documentation to be generated and a schedule of technical reviews to assess the progress achieved in each project milestone.

Therefore, Systems Engineering helps to ensure that the system is correctly developed from the beginning, minimizing risks and anticipating problems that may arise.

Modeling, Systems Engineering, and Project Management for Astronomy $\mathrm{VI}$, edited by George Z. Angeli, Philippe Dierickx, Proc. of SPIE Vol. 9150, 915026

(C) 2014 SPIE $\cdot$ CCC code: 0277-786X/14/\$18 - doi: 10.1117/12.2055916 
The main tasks that should be carried out by the System Engineer are summarized as follows:

- Implement the requirements engineering, which aims to ensure that the high-level requirements are correctly interpreting user needs and including all environmental and external interface constrains and ensure traceability with the lower level specifications.

- Perform system analysis, resolve requirement conflicts, carry out trade-off, develop and use simulation models, analyze project risks and perform RAMS analysis.

- Define and maintain system configuration (define Product Tree and Interface Table) and manage nonconformities and configuration changes that could arise during the system development.

- Prepare and execute the Integration and Verification Plan.

- Prepare the Operation and Maintenance Plan.

During the Conceptual Phase of the MEGARA project, the MEGARA System Engineering Plan was defined considering all project phases, from conceptual design to the final instrument acceptance at GTC. The activities are reviewed at the end of each phase in order to add the needed details to the tasks to be performed in the following phase. Currently, MEGARA has already delivered the CDR documentation and is ready to enter into manufacturing. This article summarizes how system engineering has been implemented at MEGARA and the current status of the activities.

\section{MEGARA OVERVIEW}

MEGARA (Multi-Espectrógrafo en GTC de Alta Resolución para Astronomía) is an optical Integral-Field Unit (IFU) and Multi-Object Spectrograph (MOS) designed for the GTC 10.4m telescope in La Palma.

MEGARA offers two IFU-type modes with two different bundles, one covering $12.5 \operatorname{arcsec} \times 11.3 \operatorname{arcsec}$ with a spaxel size of 0.62 arcsec (Large Compact Bundle; LCB, which makes use of $100 \mathrm{~mm}$-core optical fibers) and another one covering 8.5 arcsec $x 6.7$ arcsec with a spaxel size of $0.42 \operatorname{arcsec}$ (Small Compact Bundle; SCB, with 70mm-core fibers). The MEGARA MOS mode will allow observing up to 100 objects in a region of 3.5 arcmin $\times 3.5$ arcmin around the two IFU bundles. Each of the MEGARA MOS positioners can place a mini-bundle of 7 fibers ( 0.62 "/fiber) covering an area of $1.6 "$ on the sky. Eight of these bundles will be devoted to the determination of the sky during the observation with the LCB IFU, so only 92 of these positioners will be available for MOS observations.

\begin{tabular}{|l|l|c|c|c|}
\hline \multicolumn{2}{|c|}{ Parameter } & LCB & SCB & MOS \\
\hline Spaxel size & $0.62 \mathrm{arcsec}$ & $0.42 \mathrm{arcsec}$ & $0.62 \mathrm{arcsec}$ \\
\hline Field of View & $12.5 \times 11.3 \mathrm{arcsec}^{2}$ & $8.5 \times 6.7 \mathrm{arcsec}^{2}$ & $3.5 \times 3.5 \mathrm{arcmin}^{2}$ \\
\hline$\Delta \lambda\left(\mathrm{EED}_{80}\right)$ & Requirement & 4 pix & $3.48 \mathrm{pix}$ & 4 pix \\
\hline$\Delta \lambda(\mathrm{FWHM})$ & Requirement & $3.6 \mathrm{pix}$ & $3.14 \mathrm{pix}$ & $3.6 \mathrm{pix}$ \\
\hline \multirow{3}{*}{$\begin{array}{l}\left(\mathrm{R}_{\mathrm{FWHM}}=\lambda / \Delta \lambda\right) \\
(\text { Requirement })\end{array}$} & LR VPHs & 6,000 & 7,000 & 6,000 \\
\cline { 2 - 5 } & MR VPHs & 12,000 & 13,500 & 12,000 \\
\cline { 2 - 5 } & HR VPHs & 18,700 & 21,500 & 18,700 \\
\hline
\end{tabular}

Table 1. Main characteristics of the MEGARA LCB and SCB IFU and MOS modes.

In MEGARA Spectrograph the optical elements are placed on an optical table. The fiber bundle(s) reach the spectrograph on the pseudo-slit, where the fibers are aligned. There are 3 pseudo slits, which can be selected to the observing position with a dedicated mechanism. The focusing mechanism is also implemented moving the pseudo slits in the optical path axis direction. Just after the pseudo slit, follows the collimator barrel that includes the rotating custommade shutter. Then, follows the pupil position, where the VPHs shall be located (a mechanism is used to select among the 11 VPHs set on the wheel). Finally, follows the camera and the cryostat, where is hosted the CCD.

The set of VPHs that MEGARA will provide is included in Table 2. 


\begin{tabular}{|c|c|c|c|c|c|c|c|}
\hline VPH Name & Setup & $\mathrm{R}_{\mathrm{FWHM}}$ & $\lambda_{1}-\lambda_{2}(\AA)$ & $\lambda \mathrm{c}(\AA)$ & $\Delta \lambda(@ \lambda \mathrm{c})(\AA)$ & $\Delta \mathrm{v}(\mathrm{km} / \mathrm{s})$ & lin res $(\AA /$ pix $)$ \\
\hline VPH405-LR & LR-U & 6028 & $3653-4386$ & 4051 & 0.672 & 50 & 0.17 \\
\hline VPH480-LR & LR-B & 6059 & $4332-5196$ & 4800 & 0.792 & 49 & 0.20 \\
\hline VPH570-LR & LR-V & 6080 & $5143-6164$ & 5695 & 0.937 & 49 & 0.23 \\
\hline VPH675-LR & LR-R & 6099 & $6094-7300$ & 6747 & 1.106 & 49 & 0.28 \\
\hline VPH799-LR & LR-I & 6110 & $7220-8646$ & 7991 & 1.308 & 49 & 0.33 \\
\hline VPH890-LR & LR-Z & 6117 & $8043-9630$ & 8900 & 1.455 & 49 & 0.36 \\
\hline VPH410-MR & MR-U & 12602 & $3917-4277$ & 4104 & 0.326 & 24 & 0.08 \\
\hline VPH443-MR & MR-UB & 12370 & $4225-4621$ & 4431 & 0.358 & 24 & 0.09 \\
\hline VPH481-MR & MR-B & 12178 & $4586-5024$ & 4814 & 0.395 & 25 & 0.10 \\
\hline VPH521-MR & MR-G & 12035 & $4963-5443$ & 5213 & 0.433 & 25 & 0.11 \\
\hline VPH567-MR & MR-V & 11916 & $5393-5919$ & 5667 & 0.476 & 25 & 0.11 \\
\hline VPH617-MR & MR-VR & 11825 & $5869-6447$ & 6170 & 0.522 & 25 & 0.13 \\
\hline VPH656-MR & MR-R & 11768 & $6241-6859$ & 6563 & 0.558 & 25 & 0.14 \\
\hline VPH712-MR & MR-RI & 11707 & $6764-7437$ & 7115 & 0.608 & 26 & 0.15 \\
\hline VPH777-MR & MR-I & 11654 & $7382-8120$ & 7767 & 0.666 & 26 & 0.17 \\
\hline VPH926-MR & MR-Z & 11638 & $8800-9686$ & 9262 & 0.796 & 26 & 0.20 \\
\hline VPH665-HR & HR-R & 18700 & $6445-6837$ & 6646 & 0.355 & 16 & 0.09 \\
\hline VPH863-HR & HR-I & 18701 & $8372-8882$ & 8634 & 0.462 & 16 & 0.12 \\
\hline
\end{tabular}

Table 2. MEGARA VPHs.

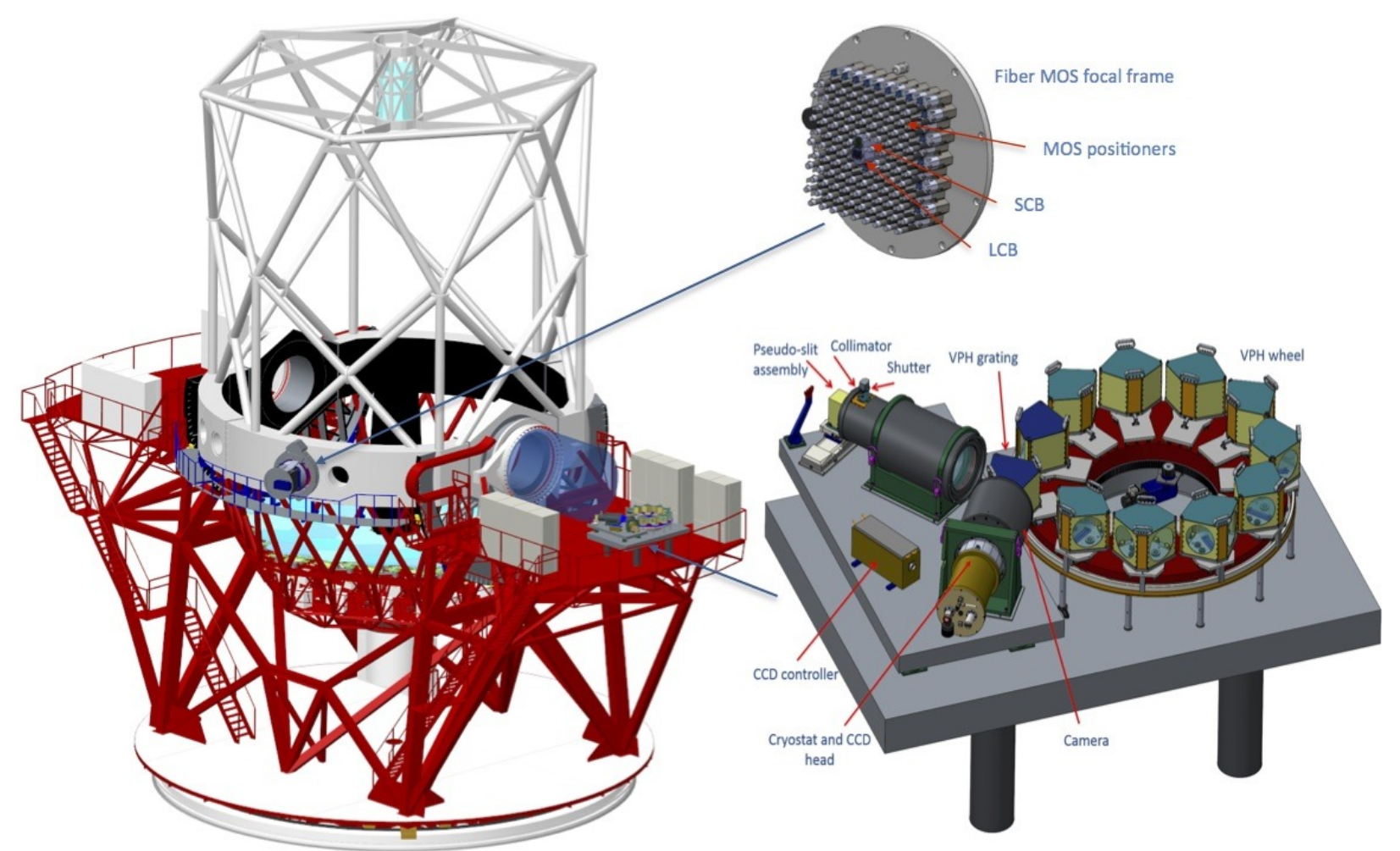

Fig. 1. Left: MEGARA at GTC. Right up: MEGARA Folded Cassegrain subsystem. Right down: MEGARA Spectrograph. 
The consortium responsible for the design and construction of the instrument includes the Universidad Complutense de Madrid (UCM, Spain) where the MEGARA Principal Investigator (Armando Gil de Paz) is located, the Instituto Nacional de Astrofísica, Óptica y Electrónica (INAOE, México), the Instituto de Astrofísica de Andalucía (IAA-CSIC, Spain), and the Universidad Politécnica de Madrid (UPM, Spain). MEGARA is being developed under contract with GRANTECAN.

\section{SYSTEM ENGINEERING TASKS}

\subsection{Requirements engineering}

Requirements engineering is the System Engineering activity devoted to develop the system requirements. This activity starts identifying the high-level requirements to iteratively generate lower level requirements during the design and decomposition of the system.

In order to define the complete set of MEGARA high-level system requirements, the top-level science performance requirements has been collected as well as the environmental, production, operation, maintenance and handling constrains that could apply to the instrument and the GTC interfaces (focal stations constrains, services to the instruments, standards to be applied, etc.) applicable to MEGARA.

During the conceptual design, two documents were generated to gather this information:

- MEGARA Functional Requirement document, where the high-level scientific requirements (taking into account both the GTC and the MEGARA scientific group high-level needs) are defined.

- MEGARA Interface to GTC Requirement document, where all GTC interfaces, environmental, operation, maintenance and handling constrain are defined.

During the preliminary and detailed design, the MEGARA System Specification document was produced to specify the solution adopted for the MEGARA instrument. This document contains the lower level specifications of the MEGARA subsystem and components. The specified solution fulfills the requirements stated in the MEGARA Functional Requirement and MEGARA Interfaces to GTC documents; traceability to both documents was implemented.

In addition, the interface control documents between the MEGARA subsystems that are developed by different groups have been defined. All requirements, specification and interface documents are now under configuration control.

Following the best engineering practices, the requirements included in the documents mentioned in the previous paragraphs have been developed fulfilling the conditions identified in the following lines:

- The requirements must be identifiable (a unique code will be assigned).

- The source of the requirement must be identified (source or parent requirement).

- Each requirement must be unique (in order to facilitate the traceability of the requirement).

- The requirement must be concise and unambiguous.

- The technical requirements of the proposed solutions (i.e., specifications) must be verifiable.

Requirements are stored and managed by the FRACTAL Configuration Management Tool (GECO), which facilitates the traceability and control of the requirements. GECO enables to establish relationships between parent and children requirements and helps the System Engineering to analyze the impact of the Configuration Changes and Nonconformities that could be raised during system development. GECO allows automatically generating requirement documents and specifications from the requirements kept in the application. 


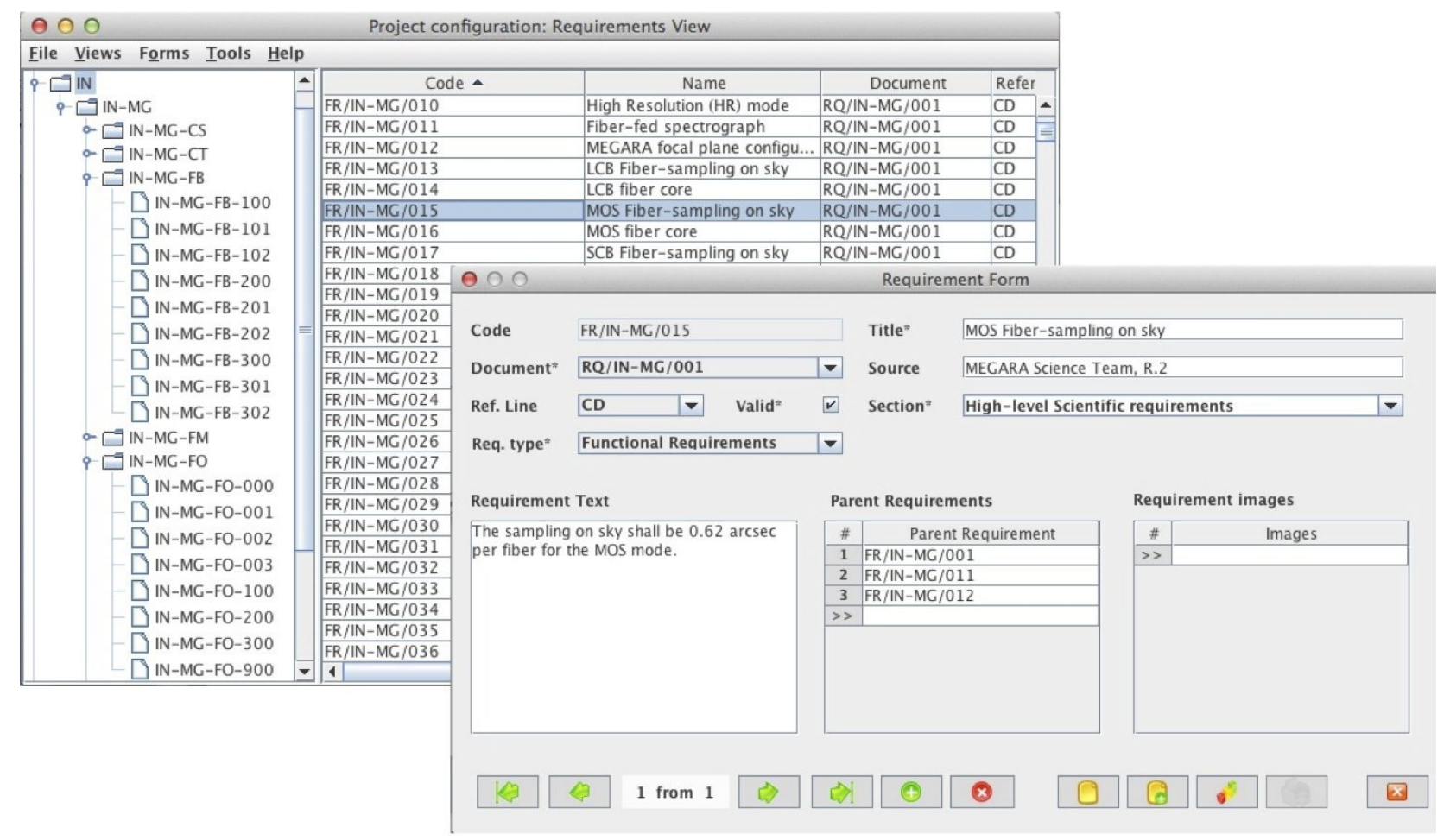

Fig. 2. MEGARA requirements list and form view at GECO.

\subsection{System technical budgets}

Technical budgets must be defined and maintained for the main physical and functional parameters of the system. The control of the technical budgets is particularly important to facilitate system integration and to guarantee that the system reaches the required performances.

In order to develop them correctly, the following issues are being taken into account:

- Budgets and tolerances must be defined for each level of the system.

- The tolerances must be reduced during the system detailed design.

- A technical budget must be defined for each physical parameter of the system (i.e., mass, power consumption, thermal dissipation, etc.).

- The technical budgets must be verified.

In MEGARA, the following technical budgets are being produced and maintained:

- Image quality to determine the optical performance of the system.

- Spectral resolution and Spectral resolution repeatability to determine spectral resolutions deviations due to static contributors (such as misalignments and manufacturing errors of the subsystems) for the spectral resolution budget and to dynamic contributors (such as the spectrograph mechanism performance) for the spectral resolution repeatability.

- Image stability to determine the stability (in position) of the spectra projected on the detector during an observation.

- Spectral alignment and Spectral alignment repeatability to determine the displacement of the estimated position of the spectra at the detector for all resolution modes (LR, MR and HR, respectively) due to static contributors 
(such as misalignments and manufacturing tolerances of the subsystems) and to dynamic contributors (such as the performance of the spectrograph mechanisms that could affect to the project spectra position).

- Throughput to determine the overall losses in the instrument since the light is received in the field lens until it reaches the detector.

- Flux homogeneity to determine the differences in flux between fibers (due to manufacturing and misalignments errors) in order to ensure a proper relative-flux calibration and cosmetics.

- Mass to identify the masses of all MEGARA components.

- Power consumption to identify the power consumption of the applicable components.

- Glycol water consumption to identify the glycol water consumption of the applicable components

- Thermal dissipation to identify the thermal dissipation of the active components.

- Reliability to identify the mean time between failures (MTBF) and mean time to repair (MTTR) of the applicable components.

\begin{tabular}{|c|c|c|c|c|c|c|c|c|c|c|}
\hline \multirow[t]{2}{*}{ ITEM } & \multicolumn{2}{|c|}{ LR } & \multicolumn{2}{|c|}{ MR } & \multicolumn{2}{|c|}{ HR } & \multirow[b]{2}{*}{ ITEM } & \multirow[b]{2}{*}{ LCB mode } & \multirow[b]{2}{*}{ MOS mode } & \multirow[b]{2}{*}{ SCB mode } \\
\hline & $\begin{array}{l}\text { Spatial } \\
\text { y-axis }\end{array}$ & $\begin{array}{c}\text { Spectral } \\
\mathbf{x} \text {-axis }\end{array}$ & $\begin{array}{l}\text { Spatial } \\
\text { y-axis }\end{array}$ & $\begin{array}{c}\text { Spectral } \\
\mathbf{x} \text {-axis }\end{array}$ & $\begin{array}{l}\text { Spatial } \\
\text { y-axis }\end{array}$ & $\begin{array}{l}\text { Spectral } \\
\mathbf{x} \text {-axis }\end{array}$ & & & & \\
\hline \multirow{3}{*}{$\begin{array}{l}\text { Pseudo slit alignment errors } \\
\begin{array}{l}\text { Collimator and camera optical } \\
\text { axis error }\end{array}\end{array}$} & \pm 3.3 & \pm 10 & \pm 3.3 & \pm 10 & \pm 3.3 & \pm 10 & Microholes position & $\pm 5 \mu \mathrm{m}$ & $\pm 5 \mu \mathrm{m}$ & $\pm 5 \mu \mathrm{m}$ \\
\hline & \multirow{2}{*}{ \pm 17} & \multirow{2}{*}{ \pm 17} & \multirow{2}{*}{ \pm 17} & \multirow{2}{*}{ \pm 17} & \multirow{2}{*}{ \pm 17} & \multirow{2}{*}{ \pm 17} & Microholes diameter (fiber centering) & $\pm 3.5 \mu \mathrm{m}$ & $\pm 3.5 \mu \mathrm{m}$ & $\pm 3.5 \mu \mathrm{m}$ \\
\hline & & & & & & & Microlens position & $\pm 5 \mu \mathrm{m}$ & $\pm 5 \mu \mathrm{m}$ & $\pm 5 \mu \mathrm{m}$ \\
\hline Camera-Coll. angle & - & \pm 22 & - & \pm 21 & - & \pm 20 & Microlens diameter & $\pm 2 \mu \mathrm{m}$ & $\pm 2 \mu \mathrm{m}$ & $\pm 2 \mu \mathrm{m}$ \\
\hline VPH lines per mm tolerance & - & \pm 60 & - & \pm 60 & - & \pm 74 & Microlens - fiber assembling and gluing & $\pm 5 \mu \mathrm{m}$ & $\pm 5 \mu \mathrm{m}$ & $\pm 5 \mu \mathrm{m}$ \\
\hline $\begin{array}{l}\text { Grating optics manufacturing } \\
\text { and assembly tolerance }\end{array}$ & - & \pm 10 & - & \pm 20 & - & \pm 20 & Support frame assembling errors (static) & - & $\pm 1 \mu \mathrm{m}$ & - \\
\hline Grating alignment tolerance & \pm 1 & \pm 4 & \pm 1 & \pm 3 & \pm 1 & \pm 2 & MOS minibundles dynamic errors & - & $\pm 4.7 \mu \mathrm{m}$ & - \\
\hline Detector alignment tolerance & \pm 6.6 & \pm 6.6 & \pm 6.6 & \pm 6.6 & \pm 6.6 & \pm 6.6 & TOTAL & $\pm 9.55 \mu \mathrm{m}$ & $\pm 10.69 \mu \mathrm{m}$ & $\pm 9.55 \mu \mathrm{m}$ \\
\hline TOTAL (pixels) & \pm 19 & \pm 68 & \pm 19 & \pm 70 & \pm 19 & \pm 82 & Requirement & $\pm 10 \mu \mathrm{m}$ & $\pm 10 \mu \mathrm{m}$ & $\pm 8 \mu \mathrm{m}$ \\
\hline Requirement (pixels) & \pm 900 & \pm 100 & \pm 900 & \pm 100 & \pm 900 & \pm 100 & & & & \\
\hline
\end{tabular}

Fig. 3. Left: Spectral alignment error budget. Right: Flux homogeneity error budget.

\subsection{System analysis}

Different system analyses will be carried out during the complete system life cycle and at different level of decomposition of the system.

Functional analysis to study the functions that must be performed by a system and assign them to lower level and tradeoffs to evaluate several alternatives and select the best one (according to the evaluation established criteria) could be required at different phases of the project.

Project analysis to evaluate that the project is fulfilling cost, schedule and technical objectives and risk analysis to identify and assess risks that could affect to the project development must be performed regularly as part of the quality control activities of the project. For project management control, MEGARA is using the FRACTAL Project Management Tool (MANATEE). The outputs of these analyses are included at MANATEE and at the MEGARA Project Management Plan.

System performance analysis must be done to evaluate the system performance and to assess the manufacturing and integration tolerances assigned to the different components. As mention in section 3.2, image quality, spectral resolution and spectral resolution repeatability, flux homogeneity, image stability, spectral alignment and spectral alignment repeatability analysis and throughput analyses have been performed to produce the system technical budgets. 
The Reliability, Availability, Maintainability and Safety (RAMS) analyses have been also performed to ensure that MEGARA design meets the availability, reliability, maintainability and safety requirements imposed to the system.

- Maintainability Analysis analyzes how to maintain the system and to minimize and facilitate its maintenance during the operation phase.

- Handling and Transport Analysis ensures that the handling and transport constrains (as stated by GRANTECAN) are compiled and establishes the corresponding requirements in the MEGARA Interface to GTC document.

- Failure Analysis (FMECA) identifies and evaluates the potential failure modes of the system, subsystems and components.

- Reliability Analysis estimates the percentage of time that the system could be unavailable taken into account the failure modes identified at the FMECA.

- Spare analysis provides a recommended spare parts list taken into account the outputs from the FMECA's and reliability analyses.

- Safety Analysis identifies and assesses the hazards that could happen to the persons that are involved in the use and maintenance of the system.

Other important point to be mention is the use of simulation models, which provide support not only during the design phase but also during the verification of the system. These models are mainly used to facilitate trade-offs (e.g., to evaluate solution performances or the feasibility of the proposed solutions) and in the maintainability analysis (e.g., study access to the different components, analyze envelopes, etc.).

The following simulation models have been generated and being used at MEGARA:

- Optical Model (Zemax)

- Mechanical and thermal model (Ansys)

- 3D dimensional model (Pro-Engineer)

- Control System Simulator.

Additionally, a Fiber MOS positioner prototype to demonstrate manufacturability and a fiber minibundle prototype to perform preliminary measurement of the fibers transmission and FRD have been produced.
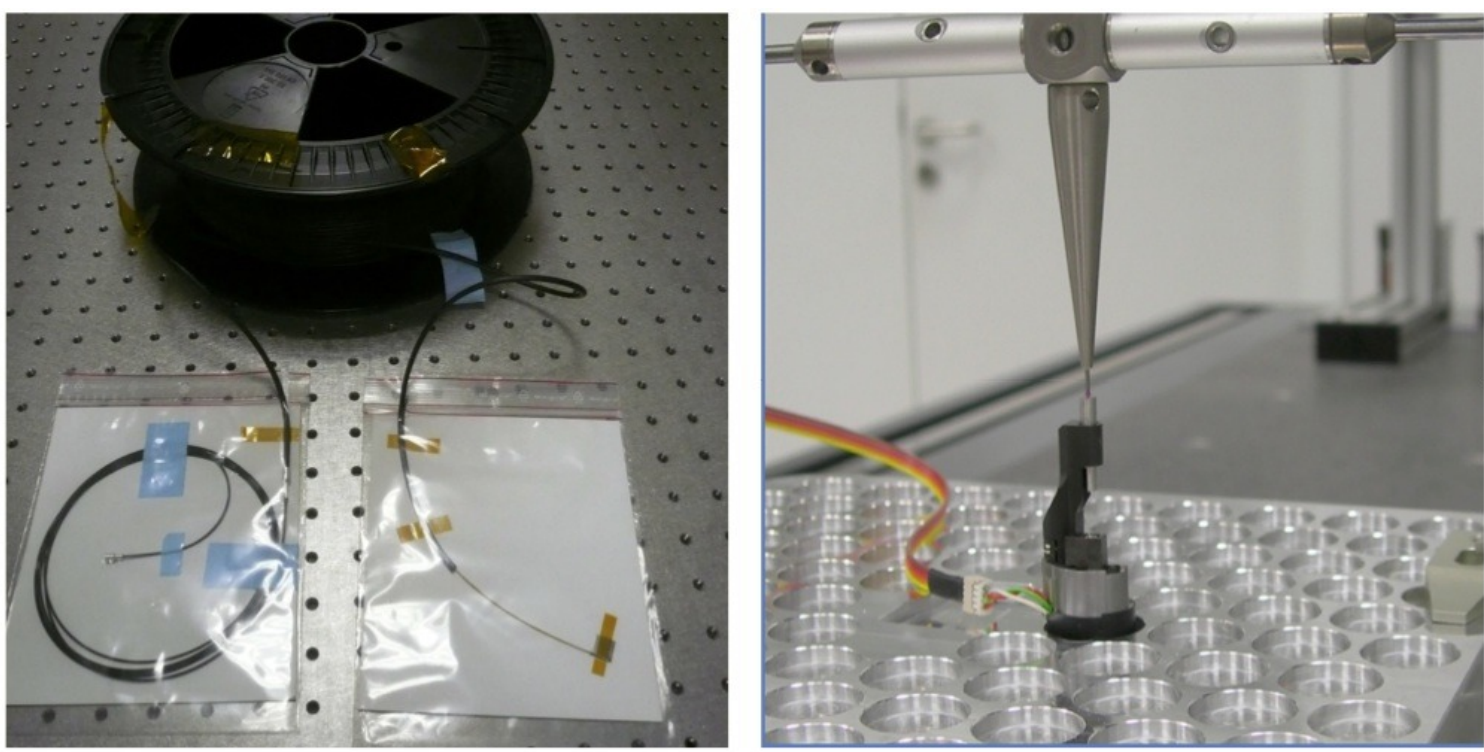

Fig. 4. Left: MEGARA fiber minibundle prototype. Right: Fiber MOS positioner at the measuring device. 


\subsection{Product tree}

The Product Tree is the hierarchical breakdown of a system into low-level elements that fully define the system. The goal is to reach a level of division into discrete elements that can be produced independently. The Product Tree is produced as result of the design process.

Each element of the Product Tree must have a unique code. At MEGARA, this code is used for coding other elements such as drawings, requirements, interfaces, etc. The Product Tree code has been defined using the GTC configuration codification rules to facilitate later the integration of the instrument in the GTC system.

The Product Tree elements are registered in GECO, from where they can be exported to an excel sheet.

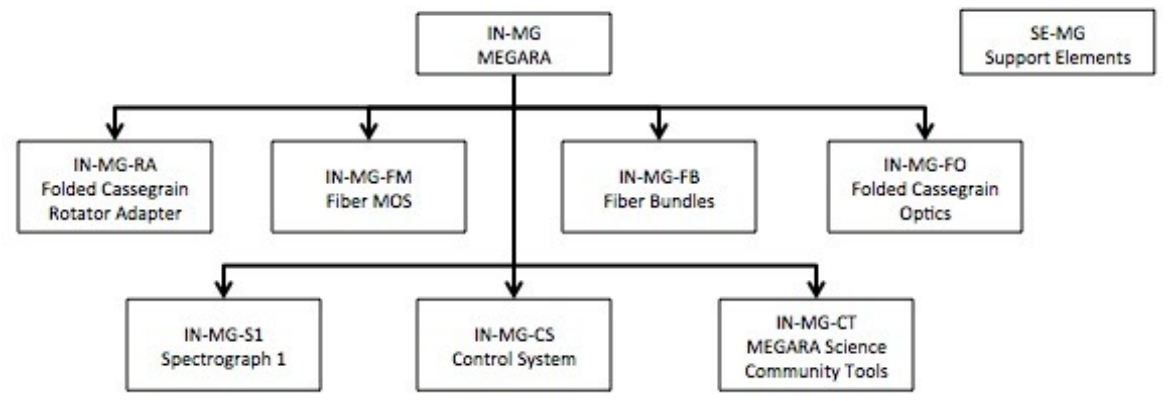

Fig. 5. MEGARA Product Tree elements at the first level.

\begin{tabular}{|c|c|c|}
\hline Code & Name & Description \\
\hline IN-MG-S1-500 & Camera & $\begin{array}{l}\text { The Camera includes the Optical and Opto-mechanical components to conduct } \\
\text { the light from the pupil position to the Detector. }\end{array}$ \\
\hline IN-MG-S1-510 & Camera Optics & The Camera Optics include the Camera Optical components. \\
\hline IN-MG-S1-511 & CAM-D1 lens & CAM-D1/CAM-D2 doublet \\
\hline IN-MG-S1-512 & CAM-D2 lens & CAM-D1/CAM-D2 doublet \\
\hline IN-MG-S1-513 & CAM-D3 lens & CAM-D3/CAM-D4 doublet \\
\hline IN-MG-S1-514 & CAM-D4 lens & CAM-D3/CAM-D4 doublet \\
\hline IN-MG-S1-515 & CAM-S5 lens & \\
\hline IN-MG-S1-516 & CAM-S6 lens & \\
\hline IN-MG-S1-517 & CAM-S7 lens & \\
\hline IN-MG-S1-520 & Camera Barrel & The Camera Barrel refers to the barrel that supports the Camera Optics elements. \\
\hline IN-MG-S1-521 & Camera Outer Barrel Subassembly & \\
\hline IN-MG-S1-521-001 & Camera outer barrel & \\
\hline IN-MG-S1-521-002 & Camera titanium flexure & $2 \mathrm{x}$ \\
\hline IN-MG-S1-521-003 & M6x14 Stainless Steel cap screw & 16 x M6x14 Stainless Steel cap screw - McMaster (92290A320) \\
\hline IN-MG-S1-521-004 & M4x16 Stainless Steel cap screw & 32 x M4x16 Stainless Steel cap screw - McMaster (92290A154) \\
\hline IN-MG-S1-521-005 & M4x0.7 Stainless Steel Hex Locknut & 32 x M4x0.7 Stainless Steel Hex Locknut - McMaster (94205A230) \\
\hline IN-MG-S1-521-006 & M4x8 Stainless Steel cap screw & 32 x M4x8 Stainless Steel cap screw - McMaster (92290A140) \\
\hline IN-MG-S1-521-007 & M5x25 Stainless Steel cap screw & 16 x M5x25 Stainless Steel cap screw - McMaster (92290A252) \\
\hline IN-MG-S1-521-008 & Camera outer barrel final ring & \\
\hline IN-MG-S1-521-009 & M5x14 Stainless Steel cap screw & 16 x M5x14 Stainless Steel cap screw - McMaster (92290A230) \\
\hline IN-MG-S1-521-010 & Camera outer barrel initial ring & \\
\hline IN-MG-S1-522 & Camera Inner Barrel Subassembly & \\
\hline IN-MG-S1-522-001 & Camera inner barrel ring & \\
\hline IN-MG-S1-522-002 & Camera inner barrel & \\
\hline IN-MG-S1-522-003 & Camera inner barrel flange & \\
\hline IN-MG-S1-522-004 & M4x16 Stainless Steel cap screw & 32 x M4x16 Stainless Steel cap screw - McMaster (92290A154) \\
\hline IN-MG-S1-522-005 & M3x6 Stainless Steel cap screw & 12 x M3x6 Stainless Steel cap screw - McMaster (92290A111) \\
\hline
\end{tabular}

Fig. 6. MEGARA Product Tree elements detailed list partial view. 


\subsection{Interfaces}

An interface is defined as the boundary between two subsystems or components (hardware or software) that are responsibility of two different groups of engineers (or contractors) that work separately.

The system interfaces may be external (with other adjoining systems) or internal (between subsystems). As mentioned in section 3.1, the MEGARA external interfaces (with GTC) are included in the MEGARA Interface to GTC Requirement document.

The internal interfaces must be identified and defined during the design process. The definition of the interfaces includes establishing and maintaining the interface table, defining the interface requirements (i.e., elaborating requirements documents for each identified interface) and maintaining interface control (as part of the configuration control).

The interfaces must be defined between physical components with the aim to minimize the amount of data that must be exchanged between components. This is particularly important from two points of view: to avoid complicating the communication between working groups and to facilitate system integration.

\begin{tabular}{|c|c|c|c|c|}
\hline Code & Element1 & Element2 & Name & Description \\
\hline IN-MG-S1-000/IN-MG-S1-400 & IN-MG-S1-000 & IN-MG-S1-400 & $\begin{array}{l}\text { Optical bench - } \\
\text { Spectral Subsystem }\end{array}$ & $\begin{array}{l}\text { INT/IN-MG-S1-000/IN-MG-S1-470/001 Support Structure - } \\
\text { Spectral subsystem mechanism } \\
\text { DR/IN-MG-S1/002 MEGARA Main subsystems positions at the } \\
\text { optical bench } \\
\text { DR/IN-MG-S1-400/001 MEGARA Spectral subsystem } \\
\text { DR/IN-MG-S1/006 MEGARA Optical bench - Wheel interface } \\
\text { DR/IN-MG-S1-479/000 MEGARA Insertion mechanism } \\
\text { DR/IN-MG-S1/007 MEGARA Optical bench - Insertion } \\
\text { mechanism interface }\end{array}$ \\
\hline IN-MG-S1-000/IN-MG-S1-700 & IN-MG-S1-000 & IN-MG-S1-700 & $\begin{array}{l}\text { Optical bench - CCD } \\
\text { Controller }\end{array}$ & $\begin{array}{l}\text { INT/IN-MG-S1-000/IN-MG-S1-700/001 Support Structure - } \\
\text { CCD Controller } \\
\text { DR/IN-MG-S1/002 MEGARA Main subsystems positions at the } \\
\text { optical bench } \\
\text { DR/IN-MG-S1-700/000 MEGARA CCD Controller } \\
\text { DR/IN-MG-S1/010 MEGARA Optical bench - CCD Controller } \\
\text { interface }\end{array}$ \\
\hline IN-MG-S1-040/IN-MG-S1-500 & IN-MG-S1-040 & IN-MG-S1-500 & $\begin{array}{l}\text { Camera-Cryostat } \\
\text { Support Structure - } \\
\text { Camera }\end{array}$ & $\begin{array}{l}\text { INT/IN-MG-S1-040/IN-MG-S1-500/001 Camera-Cryostat } \\
\text { Support Structure - Camera } \\
\text { DR/IN-MG-S1-520/000 MEGARA Camera subassembly } \\
\text { DR/IN-MG-S1-040/000 MEGARA Camera-Cryostat Support } \\
\text { Structure } \\
\text { DR/IN-MG-S1/008 MEGARA Camera-Cryostat Support } \\
\text { Structure, Camera and Cryostat interface }\end{array}$ \\
\hline IN-MG-S1-200/IN-MG-S1-300 & IN-MG-S1-200 & IN-MG-S1-300 & Shutter - Collimator & $\begin{array}{l}\text { INT/IN-MG-S1-200/IN-MG-S1-300/001 Shutter - Collimator } \\
\text { DR/IN-MG-S1-210/000 MEGARA Shutter mechanism assembly } \\
\text { DR/IN-MG-S1/004 MEGARA Shutter - Collimator interface }\end{array}$ \\
\hline IN-MG-S1-400/IN-MG-S1-400 & IN-MG-S1-400 & IN-MG-S1-400 & $\begin{array}{l}\text { VPH Optics - VPH } \\
\text { Opto-mechanics }\end{array}$ & $\begin{array}{l}\text { INT/IN-MG-S1-400/IN-MG-S1-400/001 VPH Optics - VPH } \\
\text { Optomechanics } \\
\text { DR/IN-MG-S1-412/000 LR Opto-mechanics } \\
\text { DR/IN-MG-S1-432/000 MR Opto-mechanics } \\
\text { DR/IN-MG-S1-466/000 HR Opto-mechanics }\end{array}$ \\
\hline
\end{tabular}

Fig. 7. MEGARA Interface table partial view.

\subsection{System verification}

The system verification must include all activities that will be performed to ensure that the final system meets the initial high-level requirements.

MEGARA System and subsystems acceptance will be carried out at different levels. The subsystems will be firstly accepted at factory and, then, at the MEGARA integration laboratory (LICA, Laboratorio de Instrumentatión Avanzada), which is located at the Universidad Complutense de Madrid (UCM). The whole system will be also firstly integrated and accepted at LICA and, finally, at GTC. 


\subsubsection{Verification matrices}

In order to accept the system, subsystems and components, the verification matrix of each element must be prepared including all element requirements and identifying the corresponding acceptance activities.

GECO facilitates the generation of the verification matrices by exporting the requirements of the selected elements to the matrix and also allows exporting the matrices to an excel table to be attached to the corresponding verification documents.

At the verification matrix, the following issues will be defined for each requirement:

- The verification method: testing, analysis, design review or inspection for each verification milestone.

- The verification procedure to be followed for verifying the requirement.

- The verification result to state the compliance or non-compliance of the requirement (in the later case, a nonconformity will be raised and identified at the matrix).

- The verification report, if needed, to add additional details about the verification results.

The full MEGARA verification matrix shall include all high-level system requirements, subsystems and low-level elements requirements and also interface requirements.

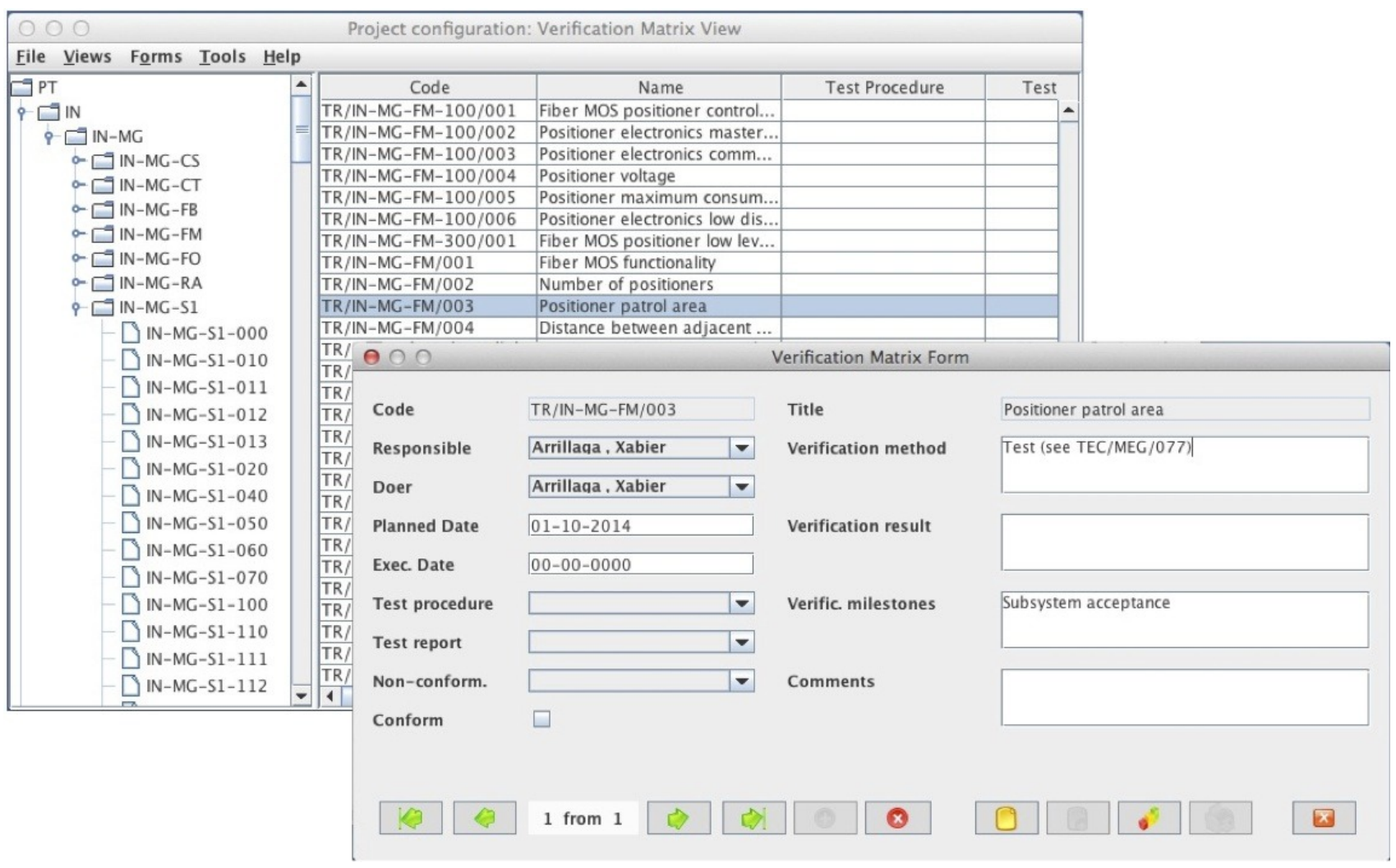

Fig. 8. GECO Verification matrix requirements list and form.

\subsubsection{Assembly, Integration and Verification (AIV) Plan}

The MEGARA AIV Plan will include the scheduling of all activities to integrate the system and verify that it fulfills the initial requirements.

During the detailed design a preliminary version of the following documents have been already produced to identify the activities that shall be performed to assembly, integrate and verify each main subsystem: 
- MEGARA. Fiber MOS acceptance tests and integration plan at AVS

- MEGARA Optical Fibers: Fibers and microlenses characterization, assembly and tests

- MEGARA Optics: Testing Plan at INAOE and CIO

- MEGARA Optics: Manufacturing Plan at INAOE and CIO

- MEGARA Cryostat Integration Plan

- MEGARA Cryostat acceptance tests at INAOE

- MEGARA Cryostat acceptance tests at LICA

- MEGARA Detector characterization: Test bench and prototype system

- MEGARA Detector Integration and Assembly Test Plan

- MEGARA Detector Characterization Test Plan

- MEGARA Spectrograph Integration Plan

- MEGARA Spectrograph Mechanisms Acceptance Tests

- MEGARA Camera and Collimator Opto-mechanics Integration and Verification Plan

- MEGARA Spectrograph. Pupil elements tests at LICA

- MEGARA Spectrograph Integration at LICA and setup

Besides the preliminary versions of the following AIV system level documents have been also produced:

- MEGARA system verification at LICA, which includes the verification activities to demonstrate that MEGARA is fulfilling the high-level requirements before being shipped to GTC.

- MEGARA Integration and Verification Plan, which summarizes all the activities that shall be done at subsystem level to accept each subsystem (as identified in the document list above), to integrate the whole instrument and to verify it at LICA.

- MEGARA Instrument Integration on Site, which contains the Integration Plan of MEGARA at GTC.

- MEGARA Commissioning plan, which contains the MEGARA Commissioning plan at GTC.

The AIV procedures must include the detailed description of the activities to be done, identifying tools, manpower, conditions to be met, the execution environment and any other relevant information that must be needed to execute and plan the activity.

The integration and verification activities to be performed at GTC during the commissioning of the instrument will be further elaborated following the GRANTECAN directions and constrains (i.e., taken into account the time and resources allocated to the MEGARA commissioning).

The MEGARA AIV Plan will be included and controlled using MANATEE, as the rest of tasks of the MEGARA Project Management Plan.

\subsection{Configuration, non-conformities and anomalies control}

All configuration elements of the system must be under configuration control. The configuration elements include the Product Tree, interfaces, requirements, specifications documents and drawings. Any change request affecting these elements shall be treated as a configuration change.

In addition, during the execution of the integration and verification activities, non-expected behaviors may appear, which would be treated as anomalies (to describe bugs or problems) or non-conformities (to describe the non-compliance of a requirement). 
Project procedures have been defined to establish how to manage configuration changes, non-conformities and anomalies at the MEGARA project.

MEGARA Systems Engineer will be responsible to implement and manage the configuration control and also will assume the Product Assurance role being responsible to analyze the anomalies and non-conformities that may occur during the integration and test phases.

All will be stored at GECO, which provides the capabilities to facilitate the analysis that must be done and to track them.

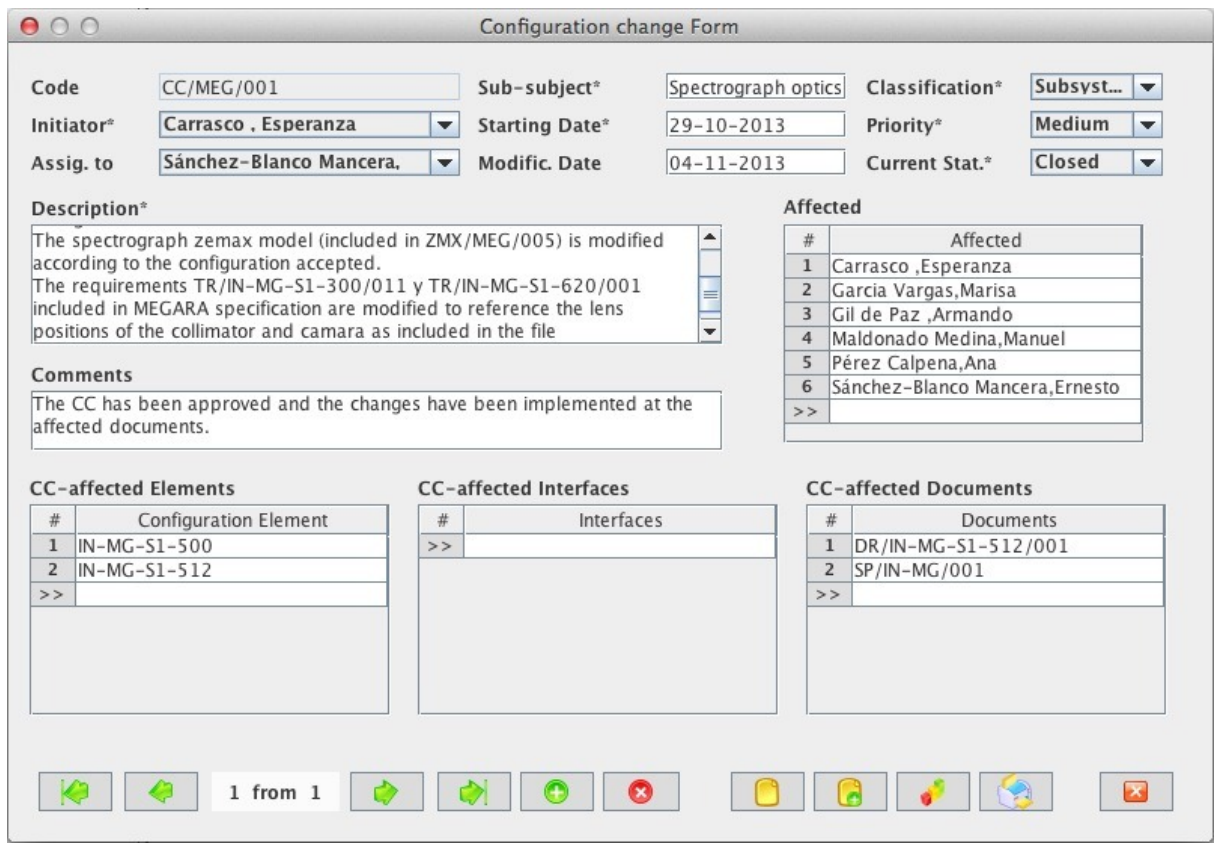

Fig. 9. GECO Configuration change form.

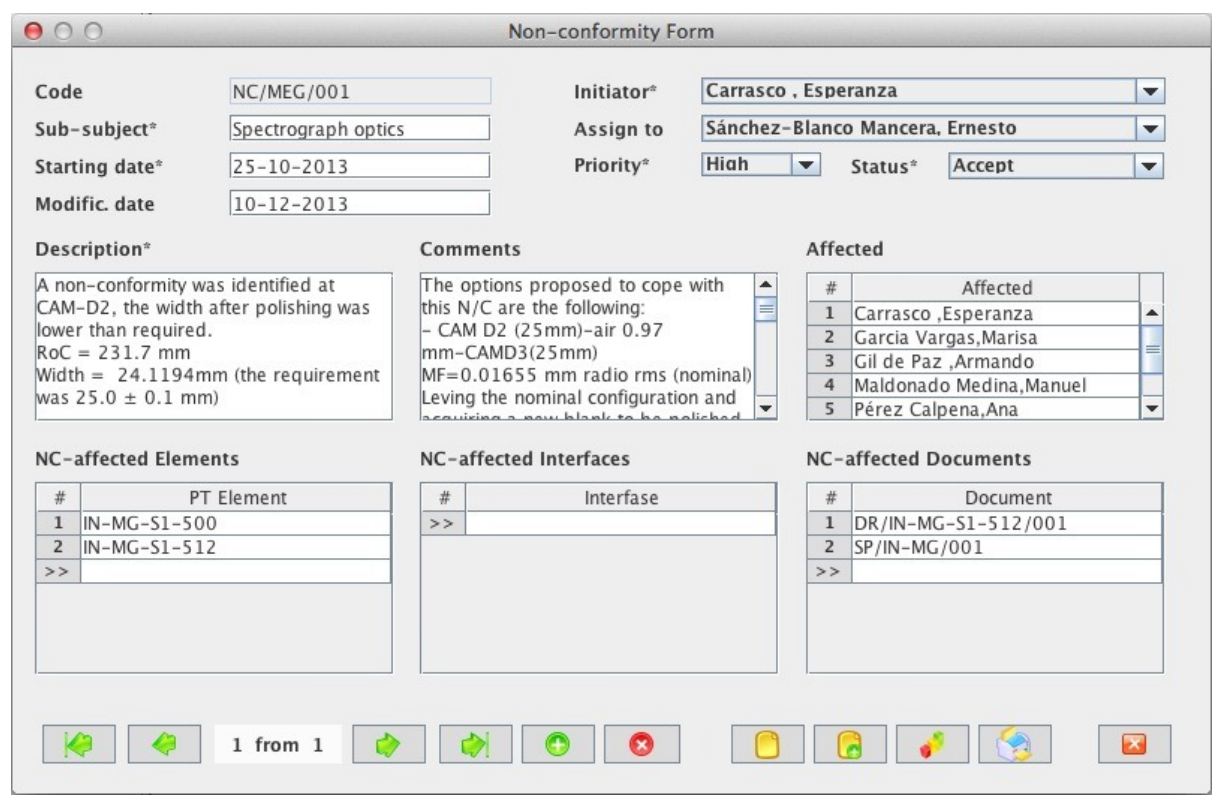

Fig. 10. GECO Non-conformity form. 


\subsection{Operation and maintenance plan}

MEGARA operation shall be part of the GTC operation plan. As a facility instrument MEGARA must define the observing modes that will offer, including science and calibration modes, and the recommended calibration plan to obtain the best scientific return of the observations to be performed. This information is already included at the MEGARA Observing Modes and MEGARA Calibration Plan documents.

The MEGARA Maintenance Plan must include all the activities that must be carried out in order to ensure that the instrument is maintained in good working conditions and is ready to be used during the night observations. The MEGARA Maintenance Plan will be generated during the following phases in agreement with the GTC Maintenance Plan and the maintenance policy stated by GRANTECAN.

\section{CONCLUSIONS}

This article describes the system engineering activities that are being performed at the MEGARA project in order to ensure that the instrument shall fulfill the scientific requirements and project constrains.

MEGARA is now entering at the manufacturing and AIV phases. The system design is fixed and under configuration control. From the system-engineering point of view, the next steps are to prepare the detailed versions of the verification matrices and AIV procedures at subsystem and system level while keeping the technical control of the project.

\section{REFERENCES}

[1] Gil de Paz, A. et al. "MEGARA: a new generation optical spectrograph for GTC", Proc. SPIE 9147, (2014)

[2] García-Vargas, M.L.et al. "Project Management for complex ground-based instruments: MEGARA plan", Proc. SPIE 9150, (2014) 